\title{
Codes Defined via Especial Matrices over the Ring and Hadamard Codes
}

\author{
Mustafa Özkan* and Figen Öke \\ (Communicated by M. Tamer KOŞAN )
}

\begin{abstract}
In this study, certain matrices are obtained using the elements of a finite chain ring. Then using these matrices as generator matrices; certain codes and their duals are obtained. Moreover relations between these codes, binary codes and Hadamard codes are explained.

Keywords: Hadamard Codes; Gray Map; Codes Over Rings.

AMS Subject Classification (2010): Primary: 94B05 ; Secondary: $94 B 15$.

*Corresponding author
\end{abstract}

\section{Introduction}

Various type of linear codes such as cyclic, constacyclic codes over the ring $\mathbb{F}_{2}+v \mathbb{F}_{2}$ where $v^{2}=1$ and $v^{2}=v$ are studied. Different type of Gray maps from these rings to Galois fields are defined and certain relations that fixed the distance between the codes on these rings and fields are established. We note that Lee distance and homogeneous distance are used over the rings $\mathbb{F}_{2}+v \mathbb{F}_{2}$ and $\mathbb{F}_{p^{k}}+u \mathbb{F}_{p^{k}}+\ldots+u^{m} \mathbb{F}_{p^{k}}$ respectively. Hadamard codes were studied before in [5]. Especially, A relation between Hadamard codes and some special codes over $\mathbb{F}_{2}+u \mathbb{F}_{2}$ was studied by M. Özkan and F. Öke in [1]. In this paper a relation between Hadamard codes and some special codes over the rings $\mathbb{F}_{2}[v] /\left\langle v^{2}-1\right\rangle$ and $\mathbb{F}_{2}[v] /\left\langle v^{2}-v\right\rangle$ are studied.

In this study, certain specifical matrices are created with lexicographically order relation on the ring $\mathbb{F}_{2}+v \mathbb{F}_{2}$ where $v^{2}=1$ and $v^{2}=v$ and then codes with $(n, 4 n, n)$-parameters are defined by using these matrices.

It is seen that the images of these codes under the suitable Gray maps are the codes with $(2 n, 4 n, n)$-parameters over the field $\mathbb{F}_{2}$. Moreover these codes are Hadamard codes. In this study the relations between cyclic, constacyclic and Hadamard codes written in the special cases are investigated.

\section{Preliminaries}

The ring $\mathbb{F}_{2}[v] /\left\langle v^{2}-1\right\rangle$ is isomorphic to the ring $\mathbb{F}_{2}+v \mathbb{F}_{2}$ where $v^{2}=1$ and the ring $\mathbb{F}_{2}[v] /\left\langle v^{2}-v\right\rangle$ is isomorphic to the ring $\mathbb{F}_{2}+v \mathbb{F}_{2}$ where $v^{2}=v$.

Let $C$ be a $(n, M, d)$ _code. It means that $C$ has the length $n$, it has $M$ elements and it's minimum distance is $d$. In the case $v^{2}=1$; The ideals of the ring $R=\mathbb{F}_{2}+v \mathbb{F}_{2}$ are $\langle 0\rangle=\{0\},\langle 1+v\rangle=\{0,1+v\}$ and $\langle v\rangle=\langle 1\rangle=R$. The property $\langle 0\rangle \subseteq\langle 1+v\rangle \subseteq\langle v\rangle \subseteq\langle 1\rangle=R$ is satisfied for these idaeals and then $R$ is a local ring.

The Lee weight $w_{L_{R}}(x)$ of $x \in R$ is given by

This article is the written version of author's plenary talk delivered on August 16-19, 2016 at 5th International Eurasian Conference on Mathematical Sciences and Applications (IECMSA-2016) in Belgrade, Serbia.

Received : 03-November-2016, Accepted : 30-March-2017 


$$
w_{L_{R}}(x)=\left\{\begin{array}{lll}
0 & ; & x=0 \\
1 & ; & x=1, v \\
2 & ; & x=1+v
\end{array}\right.
$$

This extends to a weight function in $R^{n}$. If $r=\left(r_{1}, r_{2}, \ldots, r_{n}\right) \in R^{n}$ then $w_{L_{R}}(r)=\sum_{i=1}^{n} w_{L_{R}}\left(r_{i}\right)$. The distance $d_{L_{R}}(a, b)$ between any distinct vectors $a, b \in R^{n}$ is defined to be $d_{L_{R}}(a, b)=w_{L_{R}}(a-b)$. The minimum distance $d_{L_{R}}$ of $C$ is defined as $d_{L_{R}}(C)=\min \left\{d_{L_{R}}(a, b)\right\}$ for any $a, b \in C, a \neq b$. In the case $v^{2}=v$; The ideals of the ring $S=\mathbb{F}_{2}+v \mathbb{F}_{2}$ are $\langle 0\rangle=\{0\},\langle v\rangle=\{0, v\},\langle 1+v\rangle=\{0,1+v\},\langle 1\rangle=S$ the properties $\langle 0\rangle \subseteq\langle 1+v\rangle \subseteq\langle 1\rangle=S$ and $\langle 0\rangle \subseteq\langle v\rangle \subseteq\langle 1\rangle=S$ are satisfied and then $S$ is not a local ring.

The Lee weight $w_{L_{R}}(x)$ of $x \in S$ is given by

$$
w_{L_{S}}(x)= \begin{cases}0 & ; \quad x=0 \\ 1 \quad & ; x=v, 1+v \\ 2 & ; \quad x=1\end{cases}
$$

This extends to a weight function in $S^{n}$. If $s=\left(s_{1}, s_{2}, \ldots, s_{n}\right) \in S^{n}$ then $w_{L_{S}}(s)=\sum_{i=1}^{n} w_{L_{R}}\left(s_{i}\right)$. The distance $d_{L_{S}}(a, b)$ between any distinct vectors $a, b \in S^{n}$ is defined to be $d_{L_{S}}(a, b)=w_{L_{S}}(a-b)$. The minimum distance $d_{L_{S}}$ of $C$ is defined as $d_{L_{S}}(C)=\min \left\{d_{L_{S}}(a, b)\right\}$ for any $a, b \in C, a \neq b$. Let $C$ be a code over $\mathbb{F}_{2}$ of length $n$ and let $c=\left(c_{1}, c_{2}, \ldots, c_{n}\right)$ be a codeword of $C$.

The Hamming weight of $C$ is defined as

$$
w_{H}(c)=\sum_{i=1}^{n} w_{H}\left(c_{i}\right)
$$

where $w_{H}\left(c_{i}\right)=1$ if $c_{i}=1$ and $w_{H}\left(c_{i}\right)=0$ if $c_{i}=0$. The minimum Hamming distance of $C$ is defined as $d_{H}=\min \left\{d_{H}\left(c, c^{\prime}\right)\right\}$ for any $c, c^{\prime} \in C, c \neq c^{\prime}$.

A $n \times n$ matrix such that all components are -1 or 1 and $M . M^{t}=n . I$ is called Hadamard matrix. A $n \times n$ matrix is called binary normalized Hadamard matrix if it is obtained from $M_{n} n \times n$ normalized Hadamard matrix writing 0 instead of 1 and writing 1 instead of -1 . Let $A_{n}$ be binary normalization of a binary Hadamard matrix $M_{n}$. If each two rows of $A_{n}$ are orthogonal then $\frac{n}{2}$ elements are different for these rows of $A_{n}$. Think that each row of $A_{n}$ is a vector. Then it is seen that the distance of between two rows is $\frac{n}{2}$. Write each row of matrix as a vector which has length $n$. Adding themselves and their complements to back of these vectors respectively, new vectors which has $2 n$ length are obtained. Write these new vectors as a code words. If completions of these code words join to this set, it is obtained that a Hadamard code including $4 n$ elements. Thus the minimum distance of this code is $n$.

Define the Gray maps as :

$$
\begin{aligned}
\Phi_{1}: R^{n} & \rightarrow \mathbb{F}_{2}^{2 n} \\
\left(r_{1}, r_{2}, \ldots, r_{n}\right) \mapsto \Phi\left(r_{1}, r_{2}, \ldots, r_{n}\right) & =\left(a_{1}, a_{2}, \ldots, a_{n}, b_{1}, b_{2}, \ldots, b_{n}\right)
\end{aligned}
$$

where $r_{i}=a_{i}+v b_{i} \in R$ for $1 \leqslant i \leqslant n$.

$$
\begin{aligned}
\Phi_{2} & : \quad S^{n} \rightarrow \mathbb{F}_{2}^{2 n} \\
\left(s_{1}, s_{2}, \ldots, s_{n}\right) \mapsto \Phi\left(s_{1}, s_{2}, \ldots, s_{n}\right) & =\left(c_{1}, c_{2}, \ldots, c_{n}, c_{1}+d_{1}, c_{2}+d_{2}, \ldots, c_{n}+d_{n}\right)
\end{aligned}
$$

where $s_{i}=c_{i}+v d_{i} \in S$ for $1 \leqslant i \leqslant n$.

Using the Gray maps $\Phi_{1}$ and $\Phi_{2}$, the codes which have the length $n$ over the rings $\mathbb{F}_{2}+v \mathbb{F}_{2}$ where $v^{2}=1$ and $v^{2}=v$ respectively may be corresponded to binary codes which have the length $2 n$. There is a relation $d_{L_{R}}(a, b)=d_{H}\left(\Phi_{1}(a), \Phi_{1}(b)\right)$ for $a, b \in R^{n}$ between Lee distance $d_{L_{R}}$ over $R^{n}$ and Hamming distance $d_{H}$ over $\mathbb{F}_{2}^{2 n}$. There is a relation $d_{L_{S}}\left(a^{\prime}, b^{\prime}\right)=d_{H}\left(\Phi_{2}\left(a^{\prime}\right), \Phi_{2}\left(b^{\prime}\right)\right)$ for $a^{\prime}, b^{\prime} \in S^{n}$ between Lee distance $d_{L_{S}}$ over $S^{n}$ and Hamming distance $d_{H}$ over $\mathbb{F}_{2}^{2 n}$.

This means that the Gray maps $\Phi_{1}$ and $\Phi_{2}$ are isometric. 


\section{Construction}

Over the ring $R$; Choose that all elements of first row of the matrix $N_{R}{ }^{\alpha_{1}, \alpha_{2}}$ from the set $\{1\}$, choose that all elements of the other row from the set $\{0,1, v, 1+v\}$ if $\alpha_{2}=0$ and from the set $\{0,1+v\}$ if $\alpha_{1}=0$. Assume that colums of this matrix are lexicographically ordered. This matrix constructed above is a special matrix which has $\alpha_{1}+\alpha_{2}+1$ rows. Certain examples for the matrix $N_{R}{ }^{\alpha_{1}, \alpha_{2}}$ constructed above are given below :

$$
\begin{aligned}
& N_{R}^{0,0}=[1], N_{R}^{0,1}=\left[\begin{array}{cc}
1 & 1 \\
0 & 1+v
\end{array}\right], N_{R}^{0,2}=\left[\begin{array}{cccc}
1 & 1 & 1 & 1 \\
0 & 0 & 1+v & 1+v \\
0 & 1+v & 0 & 1+v
\end{array}\right], \\
& N_{R}{ }^{0,3}=\left[\begin{array}{cccccccc}
1 & 1 & 1 & 1 & 1 & 1 & 1 & 1 \\
0 & 0 & 0 & 0 & 1+v & 1+v & 1+v & 1+v \\
0 & 0 & 1+v & 1+v & 0 & 0 & 1+v & 1+v \\
0 & 1+v & 0 & 1+v & 0 & 1+v & 0 & 1+v
\end{array}\right], N_{R}{ }^{1,0}=\left[\begin{array}{cccc}
1 & 1 & 1 \\
0 & 1 & v & 1+v
\end{array}\right], \\
& N_{R}^{2,0}=\left[\begin{array}{cccccccccccccccc}
1 & 1 & 1 & 1 & 1 & 1 & 1 & 1 & 1 & 1 & 1 & 1 & 1 & 1 & 1 & 1 \\
0 & 0 & 0 & 0 & 1 & 1 & 1 & 1 & v & v & v & v & 1+v & 1+v & 1+v & 1+v \\
0 & 1 & v & 1+v & 0 & 1 & v & 1+v & 0 & 1 & v & 1+v & 0 & 1 & v & 1+v
\end{array}\right], \\
& N_{R}{ }^{1,1}=\left[\begin{array}{cccccccc}
1 & 1 & 1 & 1 & 1 & 1 & 1 & 1 \\
0 & 0 & 1 & 1 & v & v & 1+v & 1+v \\
0 & 1+v & 0 & 1+v & 0 & 1+v & 0 & 1+v
\end{array}\right]
\end{aligned}
$$

Define the code $C_{R}^{\alpha_{1}, \alpha_{2}}=\left\{\left(c_{1}, c_{2}\right) \cdot N_{R}^{\alpha_{1}, \alpha_{2}} \mid c_{1} \in R^{\alpha_{1}+1}, c_{2} \in \mathbb{F}_{2}^{\alpha_{2}}\right\}$ which has a generator matrix $N_{R}^{\alpha_{1}, \alpha_{2}}$ where $\alpha_{1}, \alpha_{2}$ are integers such that $\alpha_{1}, \alpha_{2} \geqslant 0$. The lenght of this code is $n=2^{2 \alpha_{1}+\alpha_{2}}$. Moreover ; in the case $v^{2}=1$, the parameter of the code $C_{R}^{\alpha_{1}, \alpha_{2}}$ over $\mathbb{F}_{2}+v \mathbb{F}_{2}$ is $(n, 4 n, n)$.

Over the ring $S$; Choose that all elements of first row of the matrix $N_{S}{ }^{\alpha_{1}, \alpha_{2}}$ from the set $\{1\}$, choose that all elements of the other row from the set $\{0,1, v, 1+v\}$ if $\alpha_{2}=0$ and from the set $\{0,1\}$ if $\alpha_{1}=0$. Assume that colums of this matrix are lexicographically ordered. This matrix constructed above is a special matrix which has $\alpha_{1}+\alpha_{2}+1$ rows. Certain examples for the matrix $N_{S}{ }^{\alpha_{1}, \alpha_{2}}$ constructed above are given below :

$$
\begin{aligned}
& N_{S}{ }^{0,0}=[1], N_{S}{ }^{0,1}=\left[\begin{array}{ll}
1 & 1 \\
0 & 1
\end{array}\right], N_{S}{ }^{0,2}=\left[\begin{array}{llll}
1 & 1 & 1 & 1 \\
0 & 0 & 1 & 1 \\
0 & 1 & 0 & 1
\end{array}\right], N_{S}{ }^{0,3}=\left[\begin{array}{llllllll}
1 & 1 & 1 & 1 & 1 & 1 & 1 & 1 \\
0 & 0 & 0 & 0 & 1 & 1 & 1 & 1 \\
0 & 0 & 1 & 1 & 0 & 0 & 1 & 1 \\
0 & 1 & 0 & 1 & 0 & 1 & 0 & 1
\end{array}\right], \\
& N_{S}^{1,0}=\left[\begin{array}{cccc}
1 & 1 & 1 & 1 \\
0 & 1 & v & 1+v
\end{array}\right] \text {, } \\
& N_{S}^{2,0}=\left[\begin{array}{cccccccccccccccc}
1 & 1 & 1 & 1 & 1 & 1 & 1 & 1 & 1 & 1 & 1 & 1 & 1 & 1 & 1 & 1 \\
0 & 0 & 0 & 0 & 1 & 1 & 1 & 1 & v & v & v & v & 1+v & 1+v & 1+v & 1+v \\
0 & 1 & v & 1+v & 0 & 1 & v & 1+v & 0 & 1 & v & 1+v & 0 & 1 & v & 1+v
\end{array}\right], \\
& N_{S}{ }^{1,1}=\left[\begin{array}{cccccccc}
1 & 1 & 1 & 1 & 1 & 1 & 1 & 1 \\
0 & 0 & 1 & 1 & v & v & 1+v & 1+v \\
0 & 1 & 0 & 1 & 0 & 1 & 0 & 1
\end{array}\right] \text {. }
\end{aligned}
$$

Define the code $C_{S}{ }^{\alpha_{1}, \alpha_{2}}=\left\{\left(c_{1}, c_{2}\right) \cdot N_{S}{ }^{\alpha_{1}, \alpha_{2}} \mid c_{1} \in R^{\alpha_{1}+1}, c_{2} \in \mathbb{F}_{2}^{\alpha_{2}}\right\}$ which has a generator matrix $N_{S}{ }^{\alpha_{1}, \alpha_{2}}$ where $\alpha_{1}, \alpha_{2}$ are integers such that $\alpha_{1}, \alpha_{2} \geqslant 0$. The lenght of this code is $n=2^{2 \alpha_{1}+\alpha_{2}}$. Moreover ; in the case $v^{2}=v$, the parameter of the code $C_{S}{ }^{\alpha_{1}, \alpha_{2}}$ over $\mathbb{F}_{2}+v \mathbb{F}_{2}$ is $(n, 4 n, n)$.

Theorem 3.1. Let $\Phi_{1}: R^{n} \rightarrow \mathbb{F}_{2}^{2 n}$ be the Gray map. If $C_{R}^{\alpha_{1}, \alpha_{2}}$ is a code generated by the matrix $N_{R}^{\alpha_{1}, \alpha_{2}}$ over $R$, it's image $\Phi_{1}\left(C_{R}{ }^{\alpha_{1}, \alpha_{2}}\right)$ under the Gray map is the $(2 n, 4 n, n)$ _Hadamard code over the field $\mathbb{F}_{2}$.

Proof. The code $C_{R}{ }^{\alpha_{1}, \alpha_{2}}$ generated by the matrix $N_{R}{ }^{\alpha_{1}, \alpha_{2}}$ which has dimension $\left(\alpha_{1}+\alpha_{2}+1\right) \times n$ is of the form $C_{R}{ }^{\alpha_{1}, \alpha_{2}}=\left\{\left(c_{1}, c_{2}\right) \cdot N_{R}{ }^{\alpha_{1}, \alpha_{2}} \mid c_{1} \in R^{\alpha_{1}+1}, c_{2} \in \mathbb{F}_{2}^{\alpha_{2}}\right\}$. The length of $C_{R}{ }^{\alpha_{1}, \alpha_{2}}$ is $n=2^{2 \alpha_{1}+\alpha_{2}}$. It is clear that the code $C_{R}{ }^{\alpha_{1}, \alpha_{2}} \subseteq R^{n}$ is a repetition code and it has $4 n$ elements, i.e. $C_{R}{ }^{\alpha_{1}, \alpha_{2}}$ is a $(n, 4 n, n)_{-}$code. Hence $\Phi_{1}\left(C_{R}{ }^{\alpha_{1}, \alpha_{2}}\right) \subseteq \mathbb{F}_{2}^{2 n}$ and $\Phi_{1}\left(C_{R}{ }^{\alpha_{1}, \alpha_{2}}\right)$ is a binary Hadamard code with $(2 n, 4 n, n)$ parameter.

Lemma 3.1. The dual code $\left(C_{R}{ }^{\alpha_{1}, \alpha_{2}}\right)^{\perp}$ is a $\left(n, \frac{4^{n}}{4 n}, 4\right)$ _code and it's image $\Phi_{1}\left(\left(C_{R}{ }^{\alpha_{1}, \alpha_{2}}\right)^{\perp}\right)$ under the Gray map is a $\left(2 n, \frac{4^{n}}{4 n}, 4\right)$ code, in except the case $\alpha_{1}=\alpha_{2}=0$.

Proof. The generator matrix $N_{R}^{\alpha_{1}, \alpha_{2}}$ of the code $C_{R}{ }^{\alpha_{1}, \alpha_{2}}$ is the parity-check matrix of the dual code $\left(C_{R}^{\alpha_{1}, \alpha_{2}}\right)^{\perp}$. The dual code of $\left(C_{R}{ }^{\alpha_{1}, \alpha_{2}}\right)^{\perp}$ contains elements $c$ of $R^{n}$ satisfied $N_{R}{ }^{\alpha_{1}, \alpha_{2}} \cdot c^{T}=0$. It is easily seen that the number of words satisfied this condition is $\frac{4^{n}}{4 n}$ and the minimum weight of these words is 4 . Thus $\left(C_{R}{ }^{\alpha_{1}, \alpha_{2}}\right)^{\perp}$ is $\left(n, \frac{4^{n}}{4 n}, 4\right) \_$code. Also it is seen that $\Phi_{1}\left(\left(C_{R}{ }^{\alpha_{1}, \alpha_{2}}\right)^{\perp}\right)$ has the parameter $\left(2 n, \frac{4^{n}}{4 n}, 4\right)$. 
Theorem 3.2. Let $\Phi_{2}: S^{n} \rightarrow \mathbb{F}_{2}^{2 n}$ be the Gray map. If $C_{S}{ }^{\alpha_{1}, \alpha_{2}}$ is a code generated by the matrix $N_{S}{ }^{\alpha_{1}, \alpha_{2}}$ over $S$, it's image $\Phi_{2}\left(C_{S}{ }^{\alpha_{1}, \alpha_{2}}\right)$ under the Gray map is the $(2 n, 4 n, n)$ _Hadamard code over the field $\mathbb{F}_{2}$.

Proof. The proof is clear when the ring is taken instead of in the proof of Theorem 3.1.

Lemma 3.2. The dual code $\left(C_{s}{ }^{\alpha_{1}, \alpha_{2}}\right)^{\perp}$ is a $\left(n, \frac{4^{n}}{4 n}, 4\right)$ _code and it's image $\Phi_{2}\left(\left(C_{s}{ }^{\alpha_{1}, \alpha_{2}}\right)^{\perp}\right)$ under the Gray map is a $\left(2 n, \frac{4^{n}}{4 n}, 4\right)$ code, in except the case $\alpha_{1}=\alpha_{2}=0$.

Proof. The proof is clear when the ring is taken $S$ instead of $R$ in the proof of Lemma 3.1.

\section{Cyclic codes and quasi-cyclic codes of index 2}

Let $c=\left(c_{1}, c_{2}, \ldots, c_{n}\right)$ be an element of $R^{n}$ (or $\left.S^{n}\right) . c=\left(c_{1}, c_{2}, \ldots, c_{n}\right)$ is mapped one to one with $c(x)=\sum_{i=1}^{n} c_{i} x^{i} \in$ $R[x]$ ( or $S[x])$.

Definition 4.1. Let $C_{R}{ }^{\alpha_{1}, \alpha_{2}} \subseteq R^{n}$ be a linear code $n=2^{2 \alpha_{1}+\alpha_{2}}$, and define the map

$$
\begin{gathered}
\tau_{1}: R^{n} \rightarrow R^{n} \\
\left(c_{1}, c_{2}, \ldots, c_{n}\right) \mapsto \tau_{1}\left(c_{1}, c_{2}, \ldots, c_{n}\right)=\left(c_{n}, c_{1}, \ldots, c_{n-1}\right)
\end{gathered}
$$

If $\tau_{1}\left(C_{R}{ }^{\alpha_{1}, \alpha_{2}}\right)=C_{R}{ }^{\alpha_{1}, \alpha_{2}}$ then $C_{R}^{\alpha_{1}, \alpha_{2}}$ is cyclic code over $R$.

Definition 4.2. Let $C_{S}{ }^{\alpha_{1}, \alpha_{2}} \subseteq S^{n}$ be a linear code, $n=2^{2 \alpha_{1}+\alpha_{2}}$ and define the map

$$
\begin{gathered}
\tau_{2}: S^{n} \rightarrow S^{n} \\
\left(c_{1}, c_{2}, \ldots, c_{n}\right) \mapsto \tau_{2}\left(c_{1}, c_{2}, \ldots, c_{n}\right)=\left(c_{n}, c_{1}, \ldots, c_{n-1}\right)
\end{gathered}
$$

If $\tau_{2}\left(C_{S}{ }^{\alpha_{1}, \alpha_{2}}\right)=C_{S}{ }^{\alpha_{1}, \alpha_{2}}$ then $C_{S}{ }^{\alpha_{1}, \alpha_{2}}$ is cyclic code over $S$.

Definition 4.3. Let $D^{\alpha_{1}, \alpha_{2}} \subseteq \mathbb{F}_{2}{ }^{2 n}$ be a linear code , $n=2^{2 \alpha_{1}+\alpha_{2}}$ and define the map

$$
\begin{aligned}
& \sigma^{\otimes 2}: \mathbb{F}_{2}^{2 n} \rightarrow \mathbb{F}_{2}{ }^{2 n} \\
& \left(d_{1}, d_{2}, \ldots, d_{2 n}\right) \mapsto \sigma^{\otimes 2}\left(d_{1}, d_{2}, \ldots, d_{2 n}\right)=\left(d_{n}, d_{1}, \ldots, d_{n-1}, d_{2 n}, d_{n+1}, \ldots d_{2 n-1}\right)
\end{aligned}
$$

If $\sigma^{\otimes 2}\left(D^{\alpha_{1}, \alpha_{2}}\right)=D^{\alpha_{1}, \alpha_{2}}$ then $D^{\alpha_{1}, \alpha_{2}}$ is quasi-cyclic code of index 2 over $\mathbb{F}_{2}$.

Lemma 4.1. $\sigma^{\otimes 2} \Phi_{1}=\Phi_{1} \tau_{1}$ is satisfied.

Proof. Let $c=\left(c_{1}, c_{2}, \ldots, c_{n}\right) \in R^{n}$ where $c_{i}=a_{i}+v b_{i} \in R$ for $1 \leqslant i \leqslant n$. If $\Phi_{1}(c)=\Phi_{1}\left(c_{1}, c_{2}, \ldots, c_{n}\right)=\Phi_{1}\left(a_{1}+\right.$ $\left.v b_{1}, a_{2}+v b_{2}, \ldots, a_{n}+v b_{n}\right)=\left(a_{1}, a_{2}, \ldots, a_{n}, b_{1}, b_{2}, \ldots, b_{n}\right)$ then $\sigma^{\otimes 2}\left(\Phi_{1}(c)\right)=\left(a_{n}, a_{1}, \ldots, a_{n-1}, b_{n}, b_{1}, \ldots, b_{n-1}\right)$. On the other hand, $\tau_{1}\left(c_{1}, c_{2}, \ldots, c_{n}\right)=\left(c_{n}, c_{1}, \ldots, c_{n-1}\right)$. Then $\Phi_{1} \tau_{1}(c)=\Phi_{1}\left(\tau_{1}\left(c_{1}, c_{2}, \ldots, c_{n}\right)\right)=\Phi_{1}\left(c_{n}, c_{1}, \ldots, c_{n-1}\right)=$ $\left(a_{n}, a_{1}, \ldots, a_{n-1}, b_{n}, b_{1}, \ldots, b_{n-1}\right)$.

Lemma 4.2. $\sigma^{\otimes 2} \Phi_{2}=\Phi_{2} \tau_{2}$ is satisfied.

Proof. Let $c=\left(c_{1}, c_{2}, \ldots, c_{n}\right) \in S^{n}$ where $c_{i}=a_{i}+v b_{i} \in S$ for $1 \leqslant i \leqslant n$. If $\Phi_{2}(c)=\Phi_{2}\left(c_{1}, c_{2}, \ldots, c_{n}\right)=\Phi_{2}\left(a_{1}+\right.$ $\left.v b_{1}, a_{2}+v b_{2}, \ldots, a_{n}+v b_{n}\right)=\left(a_{1}, a_{2}, \ldots, a_{n}, a_{1}+b_{1}, a_{2}+b_{2}, \ldots, a_{n}+b_{n}\right)$ then $\sigma^{\otimes 2}\left(\Phi_{2}(c)\right)=\left(a_{n}, a_{1}, \ldots, a_{n-1}, a_{n}+\right.$ $\left.b_{n}, b_{1}, \ldots, a_{n-1}+b_{n-1}\right)$. On the other hand, $\tau_{2}\left(c_{1}, c_{2}, \ldots, c_{n}\right)=\left(c_{n}, c_{1}, \ldots, c_{n-1}\right)$. Then $\Phi_{2} \tau_{2}(c)=\Phi_{2}\left(\tau_{2}\left(c_{1}, c_{2}, \ldots, c_{n}\right)\right)=$ $\Phi_{2}\left(c_{n}, c_{1}, \ldots, c_{n-1}\right)=\left(a_{n}, a_{1}, \ldots, a_{n-1}, a_{n}+b_{n}, b_{1}, \ldots, a_{n-1}+b_{n-1}\right)$.

Theorem 4.1. Hadamard codes which are obtained with $N_{R}{ }^{\alpha_{1}, \alpha_{2}}$ (or $N_{s}{ }^{\alpha_{1}, \alpha_{2}}$ ) are quasi-cyclic code of index 2 , in except the case $\alpha_{2}=0$.

Proof. It is seen that the length of the codes $C_{R}{ }^{\alpha_{1}, \alpha_{2}}$ (or $C_{S}{ }^{\alpha_{1}, \alpha_{2}}$ ) which are defined in third section by using different matrices $N_{R}^{\alpha_{1}, \alpha_{2}}$ (or $\left.N_{S}^{\alpha_{1}, \alpha_{2}}\right)$ is $n=2^{2 \alpha_{1}+\alpha_{2}}$. Therefore the length of the all codes $\Phi_{1}\left(C_{R}{ }^{\alpha_{1}, \alpha_{2}}\right)$ (or $\Phi_{2}\left(C_{S}{ }^{\alpha_{1}, \alpha_{2}}\right)$ ) over $\mathbb{F}_{2}$ is $2^{2 \alpha_{1}+\alpha_{2}+1}$ and they are equal to the Hadamard codes that are obtained by using Hadamard matrices. Thus from the Lemma 4.1. (or Lemma 4.2.) the equalitions $\sigma^{\otimes 2}\left(\Phi_{1}\left(C_{R}^{\alpha_{1}, \alpha_{2}}\right)\right)=\Phi_{1}\left(\tau_{1}\left(C_{R}^{\alpha_{1}, \alpha_{2}}\right)\right)=\Phi_{1}\left(C_{R}{ }^{\alpha_{1}, \alpha_{2}}\right)$ (or $\left.\sigma^{\otimes 2}\left(\Phi_{2}\left(C_{S}{ }^{\alpha_{1}, \alpha_{2}}\right)\right)=\Phi_{2}\left(\tau_{2}\left(C_{S}{ }^{\alpha_{1}, \alpha_{2}}\right)\right)=\Phi_{2}\left(C_{S}{ }^{\alpha_{1}, \alpha_{2}}\right)\right)$ are satisfied.

Since $\Phi_{1}$ (or $\left.\Phi_{2}\right)$ is injective, it follow that $\sigma^{\otimes 2}\left(\Phi_{1}\left(C_{R}{ }^{\alpha_{1}, \alpha_{2}}\right)\right)=\Phi_{1}\left(C_{R}{ }^{\alpha_{1}, \alpha_{2}}\right)\left(\right.$ or $\sigma^{\otimes 2}\left(\Phi_{2}\left(C_{S}^{\alpha_{1}, \alpha_{2}}\right)\right)=\Phi_{2}\left(C_{S}{ }^{\alpha_{1}, \alpha_{2}}\right)$ ). Consequently Hadamard codes $\Phi_{1}\left(C_{R}{ }^{\alpha_{1}, \alpha_{2}}\right)$ ( or $\Phi_{2}\left(C_{S}{ }^{\alpha_{1}, \alpha_{2}}\right)$ ) are quasi-cyclic code of index 2 . 
Example 4.1. Write the matrix $N_{R}^{0,1}=\left[\begin{array}{cc}1 & 1 \\ 0 & 1+v\end{array}\right]$ to define the code $C_{R}^{0,1}$. Then elements of $C_{R}{ }^{0,1}$ are of the form $c=\left(c_{1}, c_{2}\right) \cdot N_{R}^{0,1}$, where $c_{1} \in R, c_{2} \in \mathbb{F}_{2} \cdot C_{R}^{0,1}=\{00,01+v, 11,1 v, v v, v 1,1+v 1+v, 1+$ $\begin{array}{ll}v & 0\end{array} \subseteq R^{2}$. It is seen that $d_{L_{R}}\left(C_{R}{ }^{0,1}\right)=2$ and $\left|C_{R}{ }^{0,1}\right|=8$ and then this is a $(2,8,2) \_$code. Therefore $\Phi_{1}\left(C_{R}{ }^{0,1}\right)=$ $\{0000,0011,1111,1100,0101,0110,1010,1001\} \subseteq \mathbb{F}_{2}^{4}$ is a $(4,8,2) \_$Hadamard code. Let $A_{2}=\left[\begin{array}{cc}1 & 1 \\ -1 & 1\end{array}\right]$ be a normalized Hadamard matrix. Writing 0 instead of 1 and 1 instead of -1 , the vectors 00 and 10 are obtained. (Adding the complements of these vectors the new vectors $00,10,11,01$ are obtained). Then using the method given above, the new codewords $0000,0011,1111,1100,0101,0110,1010,1001$ are obtained. The code formed by these codewords is $\Phi_{1}\left(C_{R}^{0,1}\right)$ code which is a $(4,8,2)$ _Hadamard code.Moreover $\left(C_{R}{ }^{0,1}\right)^{\perp}=\{00,1+v 1+v\}$, $\Phi\left(\left(C_{R}{ }^{0,1}\right)^{\perp}\right)=\{0000,1111\} . C_{R}^{0,1}$ is a cyclic code such that the equation $\tau_{1}\left(C_{R}{ }^{0,1}\right)=C_{R}{ }^{0,1}$ is provided. Similarly $\Phi_{1}\left(C_{R}{ }^{0,1}\right)$ is quasi-cyclic code of index 2 such that the equation $\sigma^{\otimes 2}\left(\Phi_{1}\left(C_{R}{ }^{0,1}\right)\right)=\Phi_{1}\left(C_{R}{ }^{0,1}\right)$ is provided.

Example 4.2. Write the matrix $N_{S}{ }^{0,2}=\left[\begin{array}{llll}1 & 1 & 1 & 1 \\ 0 & 0 & 1 & 1 \\ 0 & 1 & 0 & 1\end{array}\right]$ to define the code $C_{S}{ }^{0,2}$. Then elements of $C_{S}{ }^{0,2}$ are of the form $c=\left(c_{1}, c_{2}\right) \cdot N_{S}^{0,2}$, where $c_{1} \in R, c_{2} \in \mathbb{F}_{2}^{2}$.

$C_{S}{ }^{0,2}=\{0000,0101,0011,0110,1111,1010,1100,1001, v v v v, v 1+v v 1+v, v v 1+v 1+v$, $v 1+v 1+v v, 1+v 1+v 1+v 1+v, 1+v v 1+v v, 1+v 1+v v v, 1+v v v 1+v\} \subseteq S^{4}$.

It is seen that $d_{L_{S}}\left(C_{S}{ }^{0,2}\right)=4$ and $\left|C_{S}{ }^{0,2}\right|=16$ and then this is a $(4,16,4)$ code.Therefore $\Phi_{2}\left(C_{S}{ }^{0,2}\right)=\{00000000,01010101,00110011,0110011011111111,10101010,11001100,10011001$,

$00001111,01011010,00111100,01101001,11110000,10100101,11000011,10010110\} \subseteq \mathbb{F}_{2}^{8}$

is a $(8,16,4)_{-}$Hadamard code. Let $A_{4}=\left[\begin{array}{cccc}1 & 1 & 1 & 1 \\ -1 & 1 & -1 & 1 \\ -1 & -1 & 1 & 1 \\ -1 & -1 & -1 & 1\end{array}\right]$ be a normalized Hadamard matrix. Writing 0 instead of 1 and 1 instead of -1 , the vectors $0000,1010,1100$ and 0110 are obtained. (Adding the complements of these vectors the new vectors $0000,1010,1100,0110,1111,0101,0011$ and 1001 are obtained). Then using the method given above, the new codewords $00000000,01010101,00110011,01100110,11111111,10101010,11001100$,

10011001, 00001111, 01011010, 00111100, 01101001,11110000, 10100101, 11000011, 10010110 are obtained. The code formed by these codewords is $\Phi_{2}\left(C_{S}{ }^{0,2}\right)$ code which is a $(8,16,4)$ _Hadamard code. Moreover $\left(C_{S}{ }^{0,2}\right)^{\perp}=$ $C_{S}{ }^{0,2} \cdot C_{S}{ }^{0,2}$ is a cyclic code such that the equation $\tau_{2}\left(C_{S}{ }^{0,2}\right)=C_{S}{ }^{0,2}$ is provided. Similarly $\Phi_{2}\left(C_{S}{ }^{0,2}\right)$ is quasi-cyclic code of index 2 such that the equation $\sigma^{\otimes 2}\left(\Phi_{2}\left(C_{S}^{0,2}\right)\right)=\Phi_{2}\left(C_{S}{ }^{0,2}\right)$ is provided.

\section{References}

[1] Özkan, M. and Öke F., A relation between Hadamard codes and some special codes over $\mathbb{F}_{2}+u \mathbb{F}_{2}$. App . Mathematics and Inf. Sci 10 (2016), no. 2, 701-704.

[2] Jian-Fa,Q.,Zhang L.N. and Zhu S.X., $(1+u)$ - cyclic and cyclic codes over the ring $\mathbb{F}_{2}+u \mathbb{F}_{2}$. Applied Mathematics Letters,19, (2006), 820-823.

[3] Zhu, S., Wang, Y. and Shi, M., Some result on cyclic codes over $\mathbb{F}_{2}+v \mathbb{F}_{2}$. IEEE Trans. Inf. Theory, 56, (2010), no.4, 1680-1684.

[4] Krotov, D. S., Z4-linear perfect codes. Diskretn. Anal. Issled. Oper,7, (2000),no.4, 78-90.

[5] Krotov,D. S., Z4-linear Hadamard and extended perfect codes. Procs. of the International Workshop on Coding and Cryptography,Paris, (2001), 329-334.

[6] Vermani, L.,R., Elemants,of Algebraic Coding Theory. Chapman Hall, India, (1996).

[7] Wolfmann J., Negacyclic and cyclic codes over Z4. IEEE Trans. Inf. Theory, 45, (1999), 2527-2532.

[8] Bonnecaze A. and Udaya P., Cyclic codes and self dual codes $\mathbb{F}_{2}+u \mathbb{F}_{2}$. IEEE Trans. Inf. Theory, 45, (1999), 1250-1255. 
[9] Özkan, M. and Öke F., On Some Special Codes Over $\mathbb{F}_{3}+v \mathbb{F}_{3}+u \mathbb{F}_{3}+u^{2} \mathbb{F}_{3}$. Mathematical Sci. and App. E-Notes 4 (2016), no.1, 40-44.

[10] Özkan, M. and Öke F., Gray images of $(1+v)$ - constacyclic codes over a particular ring. Palestine Journal of Mathematics, (accepted).

\section{Affiliations}

MUSTAFA ÖZKAN

ADDRESS: Trakya University, Dept. of Mathematics, 22030, Edirne-TURKEY.

E-MAIL: mustafaozkan@trakya.edu.tr

FİGEN ÖKE

ADDRESS: Trakya University, Dept. of Mathematics, 22030, Edirne-TURKEY.

E-MAIL: figenoke@trakya.edu.tr 